\title{
Research
}

\section{Selective Infracolic Omentecomy and Fixation Reduces Complications after Laparoscopic Assisted Peritoneal Dialysis Catheter Placement} \author{
Diethard Monbaliu, MD, PhD $^{4}$ \\ ${ }^{1}$ Dept. of Nephrology, Groeninge Hospital, Kortrijk, Belgium \\ ${ }^{2}$ Dept. of Digestive and HPB Surgery, Groeninge Hospital, Kortrijk, Belgium \\ ${ }^{3}$ Interdisciplinary Research Center, Catholic University Leuven, Campus Kortrijk, Kortrijk, Belgium \\ ${ }^{4}$ Dept. of Transplantation surgery, University Hospitals, Leuven, Belgium
}

Claudine Christiaens, MD ${ }^{1}$; Andries Ryckx, $\mathrm{MD}^{2 *}$; Hans Pottel, $\mathrm{PhD}^{3}$; Peter Doubel, MD ${ }^{1}$; Franky Vansteenkiste, $\mathrm{MD}^{2}$,

${ }^{\star}$ Correspondence to: Andries Ryckx, MD; Dept. of Digestive Surgery, Groeninge Hospital, President Kennedylaan 4, 8500 Kortrijk, Belgium ; Tel. $+32(0) 56634803$; E-mail: dandries.ryckx@gmail.com

Received: August $7^{\text {th }}, 2018$; Accepted: August 15 $5^{\text {th }}, 2018$; Published: November 19 $9^{\text {th }}, 2018$

Citation: Christiaens C, Ryckx A, Pottel H, Doubel P, Vansteenkiste F, Monbaliu D. Selective infracolic omentecomy and fixation reduces complications after laparoscopic assisted peritoneal dialysis catheter placement. Gen Surg Open A Open J. 2018; I(1): 6-11

\section{Introduction}

Peritoneal dialysis (PD) as a treatment for patients with end-stage renal disease (ESRD) provides a competitive alternative to hemodialysis (HD). Long-term catheter survival remains challenging and techniques are not standardized. Advanced laparoscopic placement with fixation and omentectomy might increase catheter survival. The goal of our study was to evaluate if selective infracolic omentectomy and fixation reduced complications after CAPD catheter placement.

\section{Materials and Methods}

A prospective database of patients with CAPD catheter placement from March 2004 to March 2015 was analyzed. All procedures were performed laparoscopically assisted and under general anesthesia by a single surgeon. 78 patients were included, there were no exclusion criteria. Statistical analysis was performed with SPSS. Fisher exact test and log-rank test with calculation of P-value was executed. P-value of $<0.05$ was considered significant.

\section{Results}

Of the 78 patients who underwent catheter placement, 53 (68\%) were males and 25 (32\%) were females. The mean age was 54 (ranged from 13 to 88 years). Selective infracolic omentectomy was performed in 32 patients if the momentum reached beyond the promontory. Non-resorbable sutures to fix the catheter were applied in 33 patients. The average duration of peritoneal dialysis was 21 months (range from ten days to 84 months). Omentectomy significantly reduced the incidence of catheter obstruction ( 3 vs. $11 \%, \mathrm{P}=0.028)$ but not of catheter dislocation ( 19 vs. $30 \%, \mathrm{P}=0.101)$. Omentectomy did not significantly increase the incidence of peritonitis ( $22 \mathrm{vs.} 31 \%, \mathrm{P}=0.133)$ or exit-site infection ( $16 \mathrm{vs} 17 \%$, $\mathrm{P}=0.238)$.

Catheter fixation with non-resorbable sutures reduced catheter dislocation ( 21 vs. $23 \%, \mathrm{P}=0.226)$ and catheter obstruction( 12 vs.17\%,P=0.223) with a significant reduction of peritonitis ( 15 vs. $29 \%, \mathrm{P}=0.044)$ and no effect on exit-site infection $(15$ vs. $17 \%, \mathrm{P}=0.251)$.

\section{Conclusion}

Laparoscopic PD catheter placement with selective omentectomy and fixation of the catheter to the abdominal wall is safe and feasible and leads to fewer complications.

Key words: peritoneal, dialysis, catheter, complications, laparoscopy, omentectomy. 


\section{INTRODUCTION}

Peritoneal dialysis (PD) as a treatment for patients with end-stage renal disease (ESRD) provides a competitive alternative to hemodialysis (HD). PD has shown a higher two-year survival rate (20-48\%) compared to HD and lower overall healthcare costs. ${ }^{1}$ Berger et al found that in a series of 400 US patients median healthcare costs over a 12-month follow-up period were $\$ 43,510$ higher. $(\$ 173,507$ vs. $\$ 129,997)$ for HD patients vs. PD patients. ${ }^{2}$ Fewer hospital visits provide better patient mobility and independence and thereby a higher patient satisfaction. ${ }^{3}$ Furthermore, PD patients have better preservation of residual kidney function and lower rates of hypertension. ${ }^{4,5}$ Peritoneal dialysis (PD) is currently considered the preferred treatment for patients with congestive heart failure, vascular access failure and as a short time bridging therapy to kidney transplantation. $^{6}$

The long-term durability of peritoneal dialysis is determined by a solid and functional peritoneal access. Complication-free access depends largely upon a meticulous catheter placement technique and rigorous attention to detail at the time of implantation. ${ }^{7}$ The International Society for Peritoneal Dialysis (ISPD) recommends that each PD center should, therefore, have a specialized team dedicated to the care of PD patients with an experienced surgeon who audits his data on a regular basis. If a catheter is placed through a laparoscopic approach, this should be a procedure under general anesthesia with perioperative management of adhesions and hernias. ${ }^{8}$ Since the advent of laparoscopy numerous implantation techniques have been described with variable outcomes. They are referred to as advanced laparoscopic techniques and consist of adhesiolysis, suture fixation, omentopexy and omentectomy, and rectus sheath tunneling as part of a laparoscopic placement procedure. ${ }^{9,10}$ The Society of American Gastrointestinal and Endoscopic Surgeons (SAGES) committee recently wrote a recommendation to evaluate these different techniques. The Society encourages a laparoscopic approach with adhesiolysis, rectus sheath tunneling, and omentopexy as the golden standard technique. According to their guideline, this procedure offers the lowest rate of postoperative PD catheter dysfunction and should be the preferred implantation technique in adults. ${ }^{11}$

Ten percent of PD catheters show primary non-function. ${ }^{12} \mathrm{Sev}-$ eral factors may be responsible for malfunction, including catheter malposition or migration, intraluminal (by fibrin strands or blood clots) and extraluminal (by omental wrapping or adhesions) obstruction. Primary non-function is one of the most leading causes of PD catheter removal.

In an attempt to improve catheter survival some authors have advocated performing omentectomy/omentopexy to prevent omental wrapping and catheter blockage. However, controversies about when and how to perform these procedures still remain. A golden-standard technique to prevent catheter blockage/malposition, peritonitis, exit-site infection and improve long-term successful access and durability are still under investigation. Generally, the use of prophylactic omentectomy in peritoneal catheter placement remains at the discretion of the surgeon. Nicholson proved in his series of 235 patients with open catheter placement that omentectomy had a better catheter survival over a two-year interval (78 vs. $50 \%, \mathrm{P}=0.0002)$ and advised routine omentectomy. ${ }^{13} \mathrm{Un}$ fortunately, most studies describe only small patient series, are not randomized, have multiple surgeons and lots of biases. ${ }^{14-24}$

If the rates of major catheter complications and peritonitis were reduced, more patients could start PD and furthermore continue PD therapy for a longer period. To achieve these lower rates, evaluation of the implantation technique is imperative. Our objective was to evaluate the impact of laparoscopic catheter fixation and omentectomy on the incidence of catheter obstruction, dislocation, peritonitis and exit site infection in PD patients.

\section{MATERIALS AND METHODS}

An analysis of a prospective database of all patients who underwent laparoscopic catheter placement from March 2004 to March 2015 was executed. All procedures were performed laparoscopically and under general anesthesia. 78 patients were included. There were no exclusion criteria, none of the PD catheters was placed in an urgent setting. We defined if patients underwent catheter fixation or omentectomy. Medical records were analyzed for complications.

Catheter dislocation was confirmed with abdominal X-ray and first managed conservatively with laxatives or enema. Exit-site infections were diagnosed if signs of redness and purulent discharge were present. Tunnel infection was described as induration or redness over the subcutaneous course of the catheter associated with tenderness, pain or abscess formation. Peritonitis was defined as clinical complaints of abdominal pain, cloudy dialysate, and leukocyte count greater than $100 \mathrm{cells} / \mu \mathrm{l}$ with more than $50 \%$ polymorphonuclear cells.

\section{Surgical Technique}

Catheter placement is done, under general anesthesia, laparoscopically. We use the Swan Neck Missouri double-cuffed peritoneal dialysis catheter (Covidien ${ }^{\circ}$ ). Prior to insufflation the insertion point of the catheter is measured, usually $4 \mathrm{~cm}$ under and paramedian left from the umbilicus.

A $15 \mathrm{mmHg}$ pneumoperitoneum is installed in the left subcostal region, through a Veress needle. A $5 \mathrm{~mm}$ trocar placed in the same region allows us to place 1 or 2 extra $5 \mathrm{~mm}$ trocars in the left flank/fossa. The abdominal cavity is inspected for adhesions, hernias and the length of the omentum is estimated. Adhesions are carefully taken down using electrocautery. Incidental herniae are fixed. If the omentum reaches the pelvis (beyond the promontory) an infracolic omentectomy is performed using the Ultracision harmonic scalpel $\left(\right.$ Ethicon $\left.^{\circ}\right)$. The omentum is extracted through the trocar incision in the left iliac fossa.

Subsequently the incision for the placement of the catheter is made; after incision of skin and subcutaneous fat, the anterior rectus sheath is incised horizontally. Rectus muscle fibers are split, and a purse string (Mersutures 0, Ethicon ${ }^{\circ}$ ) is placed on the posterior rectus sheath. A small opening is created in the posterior rectus sheath and peritoneum, and the catheter is introduced under direct laparoscopic vision. The purse string is tightened between the Dacron patch (extra-peritoneally) and the silicone bead (intraperitoneally). The anterior rectus sheet is closed using an interrupted suture (Vicryl 0 , Ethicon ${ }^{\circ}$ ). The catheter is tunneled, and extracted in the left fossa, putting the second Dacron patch in the subcutis.

Laparoscopically the catheter is fixed to the peritoneum of the abdominal wall below the umbilicus using a resorbable (Vicryl 2-0, PDS 2-0 Ethicon ${ }^{\circledR}$ ) suture, now using a non resorbable suture (Ticron 3/0 Ethicon ${ }^{\circ}$ ). The catheter is placed in the Douglas pouch. In-and outflow are tested twice by filling and emptying the abdominal cavity with $2 \mathrm{l}$ of fluid. Trocars are extracted under direct vision. All fascial defects $>1 \mathrm{~cm}$ are closed to prevent hernia formation. Skin is closed with intradermal sutures.

\section{Statistical Analysis}


Statistical analysis was performed with SPSS. Fisher-exact test was performed with calcution of P-value. P-value of $<0.05$ was considered significant. Kaplan-Meier curves were plotted and logrank- test was performed with the calculation of P-value.

\section{RESULTS}

Of the 78 patients who underwent catheter placement, 53 (68\%) were males and $25(32 \%)$ were females. The mean age was 54 (ranged from 13 to 88 years). 17 patients had previous abdominal surgery: six a laparoscopic appendectomy, two a Hartmann resection, two a hysterectomy, two a laparoscopic colectomy, two a laparoscopic cholecystectomy, one a splenectomy, one an ovariectomy and one a nefro-ureterectomy.

There was a statistically significant difference in the incidence of previous surgery between the omentectomy and non-omentectomy group (56 vs. $23 \%, \mathrm{P}<0.001$ ).

Infracolic omentectomy was performed in 32 patients. Fixation of the catheter was performed in 43 patients, 10 catheters were fixed with resorbable sutures, 33 catheters were fixed with non-resorbable sutures.

Obstruction was recorded in ten patients (11\%). Four obstructions were managed conservatively, unfortunately six patients needed revisional surgery for obstruction. All patients with an omentectomy could be managed conservatively for catheter obstruction. Dislocation of the catheter was recorded in twenty patients $(26 \%)$. Six patients were managed conservatively, fourteen needed revisional surgery.

In total twenty patients (26\%) had revisional surgery of the catheter for dislocation and obstruction of the catheter, seven of the catheters were removed at the time of revision.

There was a significant impact of omentectomy on the number of catheter obstructions (omentectomy vs. non-omentectomy; 3 vs. $11 \%, \mathrm{P}=0.028$ ), but not on the number of dislocations (omentectomy vs. non-omentectomy; 19 vs. $30 \%, \mathrm{P}=0.101$ ). Fixation with non-resorbable sutures did not reduce the incidence of catheter dislocation (fixation vs. no fixation; 21 vs $23 \%, \mathrm{P}=0.226$ ) or obstruction (fixation vs. no fixation $; 12$ vs. $17 \%, \mathrm{P}=0.223$ ). However, there was a significant reduction in the incidence of peritonitis (fixation vs. no fixation; 15 vs. $29 \%, \mathrm{P}=0.044$ ) and a reduction of exit-site infections (fixation vs. no fixation; 15 vs $17 \%$, $\mathrm{P}=0.251$ ) when catheters were fixed with non-resorbable sutures. Leakage was recorded in three patients (3\%), all patients had undergone omentectomy, two fixations with non-resorbable sutures. All leakages were managed conservatively. Omentectomy was correlated to a higher chance of peritoneal leakage ( 9 vs. $0 \%, \mathrm{P}=0.065$ ).

Overall incidence of peritonitis was $26 \%$ (20 patients). We recorded an overall peritonitis rate of 1 per 40 patient months. Omentectomy did not significantly influence the incidence of peritonitis (omentectomy vs non-omentectomy; 22 vs. $31 \%, \mathrm{P}=0.431$ ) or exit-site infection (omentectomy vs. non-omentectomy ; 16 vs. $17 \%, \mathrm{P}=1.0$ ).

Hernias were diagnosed and treated in twelve patients during follow-up: three inguinal hernias (two bilateral), five umbilical hernias (two incarcerated), two epigastric hernias and two incisional hernias. All hernias were treated by mesh hernioraphy, no recurrent hernia was recorded. Omentectomy did not impact the hernia rate, but both patients with an incisional hernia had undergone omentectomy.

One patient in the omentectomy group developed a hemoperitoneum on post-operative day 1 and was urgently revised for an omental bleeding. There were no other early postoperative complications.

Kaplan-Meier curves of catheter survival show no significant impact of omentectomy or catheter fixation on overall catheter survival (log-rank test, $\mathrm{P}=0.792$ ).

\section{DISCUSSION}

\section{Omentectomy and Infection}

The amazing capacities of the omentum to seal of abdominal infection and hollow viscus perforation are well known. The pedicled omental flap and its use for breast reconstruction and closure of surgical defects are widespread and has proven its durability and long-term benefit. ${ }^{25,26}$ Immunological omental effects are caused by the production of vascular endothelial growth factors, cytokines and other biological agents by adipocytes in the omental fat. ${ }^{27}$ Recurrent peritonitis may lead to irreversible changes of the peritoneal membrane function, resulting in permanent PD failure and primary catheter failure.

In our series, omentectomy did not significantly increase the incidence of peritonitis or exit-site infection. Fixation of the catheter significantly reduced the incidence of peritonitis and had no effect on exit-site infections. Although fixation introduces an extra foreign object (sutures) in the abdomen, paradoxically peritonitis rates decrease with fixation in our series. We have no clear explanation for this finding but can conclude that fear of infection should not convince physicians from fixating the catheter to the abdominal wall. Peritonitis causing organisms in our patients are Staphylococcus Aureus, Staphylococcus epidermidis(CNS), Klebsiella oxytoca, Corynebacterium sp, Pseudomonas aeruginosa, P. Mirabilis and S. Marcescens.

\section{Omentectomy and Obstruction}

The omentum is a well-known source of catheter obstruction and omental wrapping is a common reason for catheter removal or exchange (5-15\% of cases). ${ }^{28}$ Surgeons have developed different strategies to prevent this: omentopexy, omentectomy and omental folding have proven to reduce catheter obstruction and failure. Omentopexy was extensively studied by Ogunc et al. and with routine use of this technique the authors were able to reduce omental wrapping to $0 \%{ }^{29}$ In our series omentopexy was not performed.

Omentectomy was first described by Nicholson as an adjunct procedure for open introduction with a significant increase in catheter survival $(\mathrm{P}<0.01)(13)$. In a series of 163 pediatric patients catheter failure rate was significantly reduced with the performance of omentectomy ( $23 \%$ without omentectomy vs. $15 \%$ with omentectomy, $\mathrm{P}=0.0054) .{ }^{30}$ Some surgeons perform an omentectomy routinely, others use it more selectively when the omentum extends into the pelvis. In a series of 18 patients Goh et al. found an 83\% 1-year catheter survival $(83 \%)$ rates with an omental folding technique with plication of the omentum with silk sutures. ${ }^{31}$ In our series omentectomy significantly reduced the rate of obstruction but did not impact rates of dislocation. This finding seems logical, as the omentum is extracted at the time of surgical placement, catheter obstruction by the omentum becomes impossible. However rates in our series don't fall to zero because obstruction can also be caused by plicae sigmoidae and fimbriae ovaricae. Interestingly all of the obstructions in the omentectomy group could be managed conservatively.

\section{Obstruction and Migration}

Laparoscopic fixation helps prevent catheter migration out of the pelvis but unfortunately has an increased risk for obstructive adhesions, internal hernias, and infection. ${ }^{32}$ In a series of 19 patients who 
underwent laparoscopic catheter fixation with non-resorbable sutures to the uterus or peritoneum, fixation reduced catheter failure. However, leak rates increased because of the need for an extra laparoscopic port. ${ }^{3}$

Some authors have reported high dysfunction rates (14-12\%) and even migrating catheters after fixation. ${ }^{18,33}$ We recorded an overall obstruction rate of $11 \%$ and migration rate of $26 \%$. Obstruction was caused by omental wrapping in five patients, by wrapping in small bowel mesentery in five patients, by wrapping in sigmoid plica, ovary and hernia mesh in one patient.

Obstruction rates were higher in the non-fixated (NF) group vs. fixated (F) group (17 vs. 7\%). Migration rates were surprisingly higher in $\mathrm{F}$ vs. NF (27 vs $22 \%$ ). It is however important to mention that ten of the catheters were fixed with resorbable sutures, this is $30 \%$ of all catheter fixations. As our experience grew we noticed that these patients had high migration rates (50\%) which are caused by the fact that these sutures degrade over time and lose their fixating potential. At laparoscopy, we noted that the non-damaged peritoneum doesn't adhere so there was no remaining peritoneal tunnel to fix the catheter. If we eliminate the resorbable fixed catheters from the F group, dislocation rates drop significantly which leads to dislocation rates of $21 \%$ in the adjusted F-group vs. $23 \%$ in the NF group.

When obstruction and dislocation occurred, there was no difference in management pattern with or without fixation and most patients had to be revised.

Catheter fixation with non-resorbable sutures reduced catheter dislocation and catheter obstruction. Catheter fixation however, does not impact overall survival of the catheter.

\section{Hernias}

Circulation of peritoneal dialysate leads to increased intra-abdominal pressure and tension on the abdominal wall which causes a significant increase in the incidence of hernias in CAPD patients. Del Peso et al. examined 142 patients and found a hernia rate of $37 \%$ (primarily umbilical). In their series, all catheters were placed with an open introduction technique by a nephrologist. ${ }^{34-37}$ Hernia rates in laparoscopically placed catheters are not accurately described in the literature, leak rates vary in several studies. Schmidt et al. describe an incisional hernia rate of $6.3 \% .{ }^{16}$ ISPD and SAGES both recommend surgical repair of abdominal wall defects to prevent complications during dialysis. ${ }^{8,11}$ Laparoscopy provides a good inspection of the abdominal wall and both inguinal regions prior to catheter insertion. This gives the surgeon the opportunity to detect and/ or repair clinically occult hernia defects before starting with peritoneal dialysis and increasing abdominal pressure. In our series, we did not find any an occult hernia at the time of catheter implantation but recorded a hernia rate of $15 \%$ with five umbilical hernias.

\section{Leakage}

Peritoneal dialysate leaks occur through surgical incisions and catheter entry points. When leakage occurs it can only be managed by lowering the infusion rate and volume of dialysate or completely stopping dialysis. Leak rates vary from two to $19 \%$ with basic laparoscopic introduction and from 0 to $5 \%$ with advanced laparoscopic techniques. ${ }^{11}$ Schmidt et al. $(n=43)$ recorded a leak rate of $13 \%$ but did not perform omentectomy. ${ }^{16}$ The incidence of leaks in our cohort was 3\%. All leaks occurred in the omentectomy group but were managed conservatively. Higher leak rates after omentectomy might be caused by an increased incisional diameter at the site of endobag extraction.

All results are plotted in Figure 1 and 2, Kaplan-Meier curves are plotted in Figure 3 and 4.
Figure 1. Impact of omentectomy on complications

This figure shows a comparison between the omentectomy and no-omentectomy group concerning major complications: Leakage (1), Peritonitis (2), Obstruction (3), Dislocation (4), Exit-site infection (5).

Results are plotted by calculating percentages of complication positive patients on the total number of patients who underwent omentectomy $(n=32)$. Example: Peritonitis in the omentectomy vs. no omentectomy group (10/32 vs $10 / 46 ; 31 \mathrm{vs.} 22 \%)$

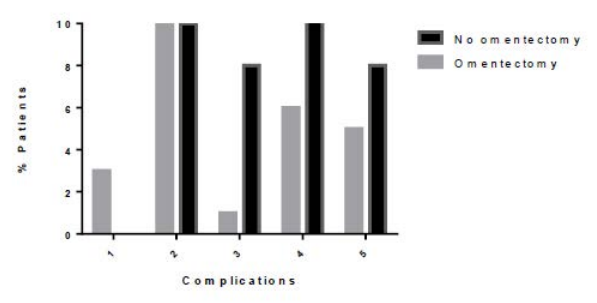

Figure 2. Impact of fixation on complications

This figure shows a comparison between the fixation non-resorbable and no-fixation group concerning major complications: Leakage (1), Peritonitis (2), Obstruction (3), Dislocation (4), Exit-site infection (5). Results are plotted by calculating percentages of complication positive patients on the total number of patients who underwent fixation $(\mathrm{n}=33)$. Example: Peritonitis in the fixation non-resorbable vs. no fixation group ( $5 / 33$ vs. $10 / 35 ; 15$ vs. $29 \%)$.

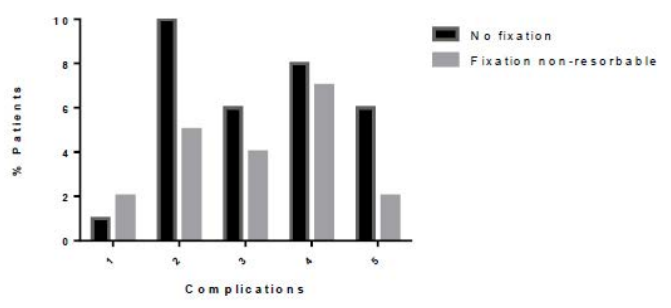

Figure 3. Kaplan-Meier curves of catheter survival

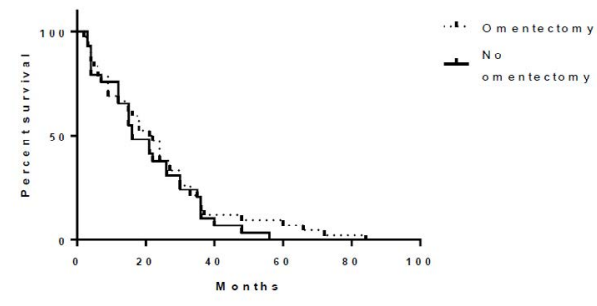

Figure 4. Kaplan-Meier curves of catheter survival

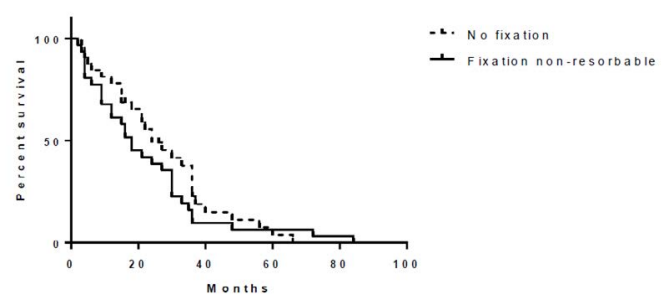

\section{Study Limitations and Strengths}

Our study has several limitations. It is a retrospective analysis of a database of a very heterogenic population. Catheters were removed because of peritonits and for tunnel infections, but some catheters were removed for benign reasons (transplantation, change to HD). This causes quite some disturbance in our data and statistical analysis because data of these patients was no longer available after catheter removal. For this reason it is also difficult to quantify the rate of catheter survival. We presumed however that data disturbances are equally distributed over the subgroups. 
Table 1. $p$-values Fisher Exact Test

\begin{tabular}{|l|c|c|c|c|c|}
\hline & Obstruction & Dislocation & Leakage & Peritonitis & $\begin{array}{c}\text { Exit-site } \\
\text { infection }\end{array}$ \\
\hline Omentectomy & 0.028 & 0.101 & 0.0652 & 0.1328 & 0.2383 \\
\hline $\begin{array}{l}\text { Fixation non- } \\
\text { resorbable }\end{array}$ & 0.2284 & 0.2266 & 0.3687 & 0.0441 & 0.2513 \\
\hline & Omentectomy $(\mathrm{n}=32)$ & \multicolumn{2}{|c|}{$\begin{array}{l}\text { No Omentectomy } \\
(\mathrm{n}=46)\end{array}$} & $p$-value \\
\hline 1.Peritonitis & $10 / 32$ & $31 \%$ & $10 / 46$ & $22 \%$ & 0.13 \\
\hline $\begin{array}{l}\text { 2.Exit-site } \\
\text { infection }\end{array}$ & $8 / 32$ & $17 \%$ & $5 / 46$ & $16 \%$ & 0.24 \\
\hline \begin{tabular}{l} 
3.Leakage \\
\hline 4.Obstruction
\end{tabular} & $3 / 32$ & $9 \%$ & $0 / 32$ & $0 \%$ & 0.06 \\
\hline 5.Dislocation & $3 / 32$ & $3 \%$ & $9 / 46$ & $11 \%$ & 0.03 \\
\hline
\end{tabular}

Our study also has some major strengths. All catheters were placed by one surgeon in a single institution with a standardised technique and a standard type of catheter with a long-term extensive follow-up. Furthermore every complication was meticulously recorded in patient files.

\section{CONCLUSION}

Patients who receive an omentectomy at time of catheter placement are less prone to obstructive catheter complications. Furthermore when obstruction occurs in these patients, a conservative management strategy is feasible. Although the omentum is considered an immunologic organ, our study proves that infracolic omentectomy has no adverse effects and does not lead to an increase in infectious complications. So considering our data, laparoscopic infracolic omentectomy is safe and feasible and should be considered as a validated technique to prevent PD catheter obstructions.

Abdominal wall fixation of the catheter is routinely performed in our institution. However fixation does not lead to a statistic significant reduction of catheter dislocation. Some have considered sutures around the catheter as an extra risk-factor for infection and small bowel obstruction (SBO). In our series no SBO was recorded, and in contrast to current opinion, fixation with non-resorbable sutures significantly reduced peritonitis.

These two interventions however have no significant impact on catheter survival.

We can conclude that a laparoscopic PD catheter placement technique is feasible and safe and has several advantages: fixation leads to less peritonitis, omentectomy leads to less catheter obstruction and routine hernia assessment is possible. Further research is necessary to determine the optimal outcomes and future guidelines for laparoscopic PD catheter placement should consider these interventions as standard technique.

\section{DISCLOSURES}

Drs. Ryckx, Christiaens, Doubel, Monbaliu, Vansteenkiste have no conflicts of interest or financial ties to disclose.

\section{REFERENCES}

1. Makkar V, Kummar M, Mahajan R, Khaira NS. Comparison of outcomes and quality of life between hemodialysis and peritoneal dialysis patients in indian esrd population. J Clin Diagn Res. 2015; 9(3): 28-31. doi: 10.7860/JCDR/2015/11472.5709

2. Berger A, Edelsberg J, Inglese GW, Bhattacharyya SK, Oster G. Cost comparison of peritoneal dialysis versus hemodialysis in end-stage renal disease. Am J Manag Care. 2009; 15(8): 509-518.

3. Juergensen E, Wuerth D, Finkelstein SH, Juergensen PH, Bekui A, Finkelstein FO. Hemodialysis and peritoneal dialysis: Patients' assessment of their satisfaction with therapy and the impact of the therapy on their lives. Clin J Am Soc Nephrol. 2006; 1: 1191-1196. doi: 10.2215/CJN.01220406

4. Mujais S, Holmes C. Modern peritoneal dialysis: Concepts and approaches. Kidney Int. 2006; 70(103): s1-s2. doi: 10.1038/sj.ki.5001909

5. Gokal R, Blake PG, Passlick-Deetjen J, Schaub TP, Prichard S, Burkart JM. What is the evidence that peritoneal dialysis is underutilized as an ESRD therapy? Sem Dial. 2002; 15(3): 149-161.

6. Shetty A. Peritoneal dialysis: Its indications and contraindications. Dial Transplant. 2000; 29: 71-77.

7. Jwo SC, Chen KS, Lee CC, Chen HY. Prospective randomized study for comparison of open surgery with laparoscopic-assisted placement of tenckhoff peritoneal dialysis catheter: A single centre experience and literature review. J Surg Res. 2010; 59: 489-496. doi: $10.1016 /$ j.jss.2008.09.008

8. Figueiredo A, Goh BL, Jenkins S, Johnson DW, Mactier R, Ramalakshmi S, et al. Clinical practice guidelines for peritoneal access. Per Dial Int. 2010; 30: 424-429. doi: 10.3747/pdi.2010.00087

9. Gajjar AH, Rhoden DH, Kathuria P, Kaul R, Udupa AD, Jennings WC. Peritoneal dialysis catheters: Laparoscopic versus traditional placement techniques and outcomes. Am J Surg. 2007; 194(6): 872876. doi: 10.1016/j.amjsurg.2007.08.038

10. Watson DI, Paterson D, Bannister K. Secure placement of peritoneal dialysis catheters using a laparoscopic technique. Surg Laparosc Endosc. 1996; 6(1): 35-37.

11. Haggerty S, Roth S, Walsh D, et al. Guidelines for laparoscopic peritoneal dialysis access surgery. Surg Endosc. 2014; 28(11): 30163045. doi: 10.1007/s00464-014-3851-9

12. Daniellson A. The controversy of peritoneal dialysis catheter placement. Per Dial Int. 2007; 27: 153-154.

13. Nicholson ML, Burton PR, Donnelly PK, Veitch PS, Walls J. The role of omentectomy in continuous ambulatory peritoneal dialysis. Per Dial Int. 1991; 11: 330-332.

14. Tsimoyiannis EC, Siakas P, Glantzounis G, et al. Laparoscopic placement of the tenckhoff catheter for peritoneal dialysis. Surg Laparosc Endosc Percutan Tech. 2000; 10: 218-221.

15. Soontrapornchai P, Simapatanapong T. Comparison of open and laparoscopic secure placement of peritoneal dialysis catheters. Surg Endosc. 2005; 19: 137-139. doi: 10.1007/s00464-004-8156-y

16. Schmidt SC, Pohle C, Langrehr JM, Schumacher G, Jacob D, Neuhaus P. Laparoscopic-assisted placement of peritoneal dialysis catheters: Implantation technique and results. J Laparoendosc $A d v$ Surg Tech A. 2007; 17(5): 596-599. doi: 10.1089/lap.2006.0162

17. Ko J, Ra W, Bae T, Lee T, Kim HH, Han HS. Two-port laparoscopic placement of a peritoneal dialysis catheter with abdominal wall fixation. Surg Today. 2009; 39: 356-358. doi: 10.1007/s00595008-3877-5

18. Bar-Zohar D, Sagie B, Lubezky N, Blum M, Klausner J, AbuAbeid S. Laparoscopic implantation of the tenckhoff catheter for the 
treatment of end-stage renal failure and congestive heart failure: Experience with the pelvic fixation technique. Isr Med Assoc J. 2006; 8: 174-178.

19. Keshvari A, Najafi I, Jafari-Javid M, Yunesian M, Chaman R, Taromlou MN. Laparoscopic peritoneal dialysis catheter implantation using a tenckhoff trocar under local anesthesia with nitrous oxide gas insufflation. Am J Surg. 2009; 197: 8-13. doi: 10.1016/j.amjsurg.2007.10.022

20. Attaluri V, Lebeis C, Brethauer S, Rosenblatt S. Advanced laparoscopic techniques significantly improve function of peritoneal dialysis catheters. J Am Coll Sur. 2010; 211: 699-704. doi: 10.1016/j.jamcollsurg.2010.08.010

21. Gotch FA, Sargent JA. A mechanistic analysis of the national cooperative dialysis study (NCDS). Kidney Int. 1985; 28: 526-534.

22. Morton MA. Distribution of intra-abdominal malignant seeding: dependency on dynamics of flow of ascitic fluid. Am J of Roentgenol Radium Ther Nucl Med. 1973; 119(1): 198-206.

23. Carmignani PC, Tessa AS, Bromley CM, Sugarbaker PH. Intraperitoneal cancer dissemination: Mechanisms of the patterns of spread. Cancer Metastasis Rev. 2003; 22(4): 465-72.

24. Verwaal VJ, van Ruth S, de Bree E, van Slooten G, van Tinteren H. 8-year follow-up of randomized trial: cytoreduction and hyperthermic intraperitoneal chemotherapy versus systemic chemotherapy in patients with peritoneal carcinomatosis of colorectal cancer. Ann Surg Oncol. 2008; 15(9): 2426-2432. doi: 10.1245/s10434-008-9966-2

25. Zaha H, Inamine S, Naito T, Nomura H. Laparoscopically harvested omental flap for immediate breast reconstruction. Am J Surg. 2006; 192(4): 556-558. doi: 10.1016/j.amjsurg.2006.06.030

26. Killeen S, Devaney A, Mannion M, Martin ST, Winter DC. Omental pedicle flaps following proctectomy: A systematic review. Col Dis. 2013; 15(11): 634-645. doi: 10.1111/codi.12394

27. Chandra A, Srivastava RK, Kashyap MP, Kumar R, Srivastava RN, Pant $\mathrm{AB}$. The anti-inflammatory and antibacterial basis of human omental defense: Selective expression of cytokines and antimicrobial peptides PLoS One. 2011; 6(5): e20446. doi: 10.1371/journal.pone.0020446
28. Kaya M, Boleken ME, Soran M, Yucesan S. Laparoscopic omental folding: A new procedure to prevent omental wraps of continuous peritoneal dialysis catheters. Eur Surg. 2012; 44(5): 345-348. doi: 10.1007/s10353-012-0161-0

29. Ogunc G, Tuncer M, Ogunc D, Yardimsever M, Ersoy F. Laparoscopic omental fixation technique versus open surgical placement of peritoneal dialysis catheters. Surg Endosc. 2003; 17: 1749-1755. doi: 10.1007/s00464-002-8586-3

30. Ladd AP, Breckler FD, Novotny NM. Impact of primary omentectomy on longevity of peritoneal dialysis catheters in children. Am J Surg. 2011; 201(3): 401-404. doi: 10.1016/j.amjsurg.2010.08.022

31. Goh YH. Omental folding: A novel laparoscopic technique for salvaging peritoneal dialysis catheters. Perit Dial Int. 28(6): 626-631.

32. Frost JH , Bagul A. A brief recap of tips and surgical manoeuvres to enhance optimal outcome of surgically placed peritoneal dialysis catheters. Int J Nephrol. 2012; 2012: 251584. doi: $10.1155 / 2012 / 251584$

33. Lu CT, Watson DI, Elias TJ, Faull RJ, Clarkson AR, Bannister KM. Laparoscopic placement of peritoneal dialysis catheters: 7-years experience. ANZ J Surg. 73(3): 109-111.

34. Del Peso G, Bajo MA, Costero O, Hevia C, Gil F, Diaz C et al. Risk factors for abdominal wall complications in peritoneal dialysis patients. Perit Dial Int. 2003; 23: 249-254.

35. Kavalakkat JP, Kumar S, Aswathaman K, Kekre NS. Continuous ambulatory peritoneal dialysis catheter placement: ls omentectomy necessary? Urol Ann. 2010; 2(3): 107-109. doi: 10.4103\%2F09747796.68858

36. Crabtree JH, Burchette RJ. Dialysis catheter embedment: Surgical considerations, expectations, and complications. Am J Surg. 2013; 206(4): 464-471. doi: 10.1016/j.amjsurg.2013.03.006

37. Crabtree JH, Burchette RJ. Effective use of laparoscopy for longterm peritoneal dialysis access. Am J Surg. 2009; 198: 135-141. doi: 10.1016/j.amjsurg.2008.10.019 